ఠ

\title{
Maintaining efficacy in the treatment of diabetic peripheral neuropathic pain: role of duloxetine
}

This article was published in the following Dove Press journal:

Diabetes, Metabolic Syndrome and Obesity:Targets and Therapy

24 December 2009

Number of times this article has been viewed

\author{
Lindsay Zilliox' \\ James W Russell ${ }^{1,2}$ \\ 'Department of Neurology, \\ Neuromuscular Division, The \\ University of Maryland School of \\ Medicine, ${ }^{2}$ VA Maryland Health Care \\ System, Baltimore, MD, USA
}

\begin{abstract}
Introduction: Neuropathy is one of the most frequent complications of diabetes. Of all the symptoms associated with diabetic neuropathy, pain has the largest impact on sleep and quality of life. In the past few years further medications have been added to the available therapies for neuropathic pain. One of these medications, duloxetine hydrochloride (duloxetine), is a balanced and potent selective serotonin and norepinephrine reuptake inhibitor.

Methods: Medline was searched from January 2005 to September 2009 using the key words duloxetine and peripheral neuropathy for clinical trials limited to human research published in English and duloxetine and pharmacology in the nervous system.

Results: Duloxetine has been shown to effectively reduce diabetic peripheral neuropathic pain compared to placebo at doses of $60 \mathrm{mg} /$ day and $120 \mathrm{mg}$ /day with minimal to moderate side effects. This effect is seen with minimal effects on glycemic control and without any clinically relevant effects on lipid control, or cardiovascular parameters. In addition, its efficacy and tolerability is comparable to other medications commonly used in the management of neuropathic pain. Furthermore, duloxetine performs favorably both in terms of quality of life and in cost utility analyses.

Discussion and conclusion: This article reviewed the issues related to management of diabetic peripheral neuropathic pain, the pharmacology and rationale for use of duloxetine, efficacy studies, and the safety and tolerability of treatment with duloxetine. Duloxetine is an acceptable initial or alternative treatment for patients with diabetic neuropathic pain.
\end{abstract}

Keywords: duloxetine, diabetic neuropathy, neuropathic pain

\section{Frequency of diabetic neuropathy}

The prevalence of diabetes mellitus in people age 20 years or greater in the United States has been estimated at $12.9 \%$. Furthermore, the prevalence of impaired fasting glucose is $25.7 \%$ and of impaired glucose tolerance is $13.8 \%$. This means that over $40 \%$ of individuals aged 20 years or older have either diabetes or pre-diabetes, and the prevalence is rising. ${ }^{1}$ Peripheral neuropathy is one of the commonest complications of diabetes. ${ }^{2}$ At least 1 in 4 patients with diabetes is affected by a distal symmetric peripheral neuropathy and neuropathic pain occurs in $7.5 \%$ to $24 \%$ of all patients with diabetes. ${ }^{2,3}$ The yearly incidence of distal symmetric polyneuropathy in diabetics is approximately $2 \%$ and the lifetime incidence of neuropathy has been estimated to be $37 \%$ to $45 \%$ for patients with type 2 diabetes and $54 \%$ to $59 \%$ for patients with type 1 diabetes. ${ }^{2,3}$ The growing prevalence of type 2 diabetes mellitus in the US and throughout the world will result in a larger number of individuals suffering from diabetic peripheral neuropathic pain.
Correspondence: James W Russel The University of Maryland School of Medicine Department of Neurology, I I 0 South Paca Street, 3S-129, Baltimore, MD 21201 , USA

$\mathrm{Tel}+\mathrm{I} 4103283100$

$\mathrm{Fax}+14103288981$

Email jrussell@som.umaryland.edu 


\section{Clinical features of neuropathy and neuropathic pain}

Diabetic peripheral neuropathy may present as a large fiber, small fiber, autonomic neuropathy, or in varying combinations of these. The neuropathy may result in pain, a dead numb feeling, prickling, or other positive or negative symptoms. The signs of diabetic neuropathy are variable but in diabetic symmetrical polyneuropathy (the most common type) include distal sensory loss affecting large or small fibers or both, and reduced reflexes.

Symptoms of diabetic neuropathic pain include deep, aching pain with superimposed burning and stabbing pain, allodynia, and hyperalgesia. ${ }^{3}$ Pain is often the complaint that motivates patients to seek medical care. However the symptoms of diabetic peripheral neuropathic pain are often difficult to treat and surveys have found that between $25 \%$ and $39 \%$ of patients may lack adequate treatment for their pain. ${ }^{3}$ This is a concern because neuropathic pain can have a major, negative impact on quality of life. For example, diabetic peripheral neuropathic pain has been reported to interfere with general activity, mood, mobility, work, social relations, sleep, leisure activities, walking ability, and enjoyment of life. ${ }^{2-4}$

Diabetic peripheral neuropathic pain is commonly attributed to a distal symmetric polyneuropathy which is associated with poor glycemic control. ${ }^{3}$ In painful diabetic neuropathy, small fibers, eg, unmyelinated (C) fibers and thinly myelinated

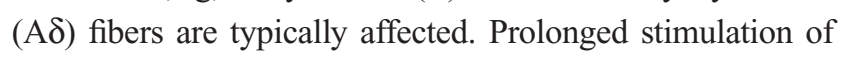
nociceptive afferents in the peripheral nervous system has been implicated in the initiation and maintenance of neuroplastic changes within the central nervous system. These changes result in persistent pain that is in part due to altered sensitivity within both the ascending and descending pain pathways between the brain and spinal cord. ${ }^{5}$ Multiple neurotransmitters are involved in these pain pathways. Dysfunction in these endogenous painmodulating circuits, which occurs in pathological pain states such as neuropathic pain, likely contributes to a state of central neuronal hyperexcitability/central sensitization. In particular, the neurotransmitters 5-hydroxytryptamine (serotonin) and norepinephrine (NE) are thought to be mediators of endogenous inhibitory pain mechanisms in the descending pain pathways. ${ }^{5,6}$ Reduced inhibition of nociceptive neurons by 5 -HT and NE in both spinal and supraspinal structures likely leads to central sensitization, which can produce spontaneous and persistent neuropathic pain. ${ }^{5}$

\section{Approach to therapy}

Currently, there are no accepted treatments to restore function to damaged nerve fibers. It has been shown that tight glycemic control can effectively slow the progression of diabetic neuropathy and delay the onset of neuropathy in type 1 diabetes, ${ }^{7}$ but this is often difficult to attain., ${ }^{3,8}$ Research trials also show that improved glycemic control in type 2 diabetic polyneuropathy is associated with a lower rate of progression to neuropathy or in prediabetes with partial reversal of the neuropathy, ${ }^{9}, 10$ Therefore, the current goal of treatment for diabetic peripheral neuropathic pain is symptomatic pain control. Widely used pharmacological treatments for neuropathic pain control include antidepressants, anticonvulsants and narcotic pain medications. Tricyclic antidepressants (TCAs) have been proposed as a first line treatment for neuropathic pain and are recommended as a drug of choice for treating diabetic peripheral neuropathic pain by the British National Formulary. ${ }^{11,12}$ Their mechanism of action is thought to be due to NE and/or serotonin reuptake inhibition within the central nervous system. However they also have other possible mechanisms of action including alpha-adrenergic blockade, sodium channel effects, and NMDA receptor antagonism. ${ }^{5}$ Clinical use of these medications is often limited by side effects such as sedation, hypotension, dry mouth, and cardiovascular abnormalities. ${ }^{8}$ Therefore, selective efficacious medications with fewer side effects would be clinically useful in the treatment of diabetic peripheral neuropathic pain.

\section{Methods}

A MEDLINE search (January 2005 to September 2009) was conducted using the key words duloxetine and peripheral neuropathy for clinical trials limited to human research published in English and duloxetine and pharmacology in the nervous system. Subsequent searches used author names or references from key manuscripts. Other searches included the Cochrane Database of Systematic Reviews, American College of Physicians Journal Club, International Pharmaceutical Abstracts, and recently published abstracts from the American Diabetes Association, American Academy of Neurology, and Peripheral Nerve Society.

\section{Overview of pharmacology and rational use of duloxetine}

Even though serotonin is a known mediator of pain, randomized trials of selective serotonin reuptake inhibitors (SSRIs) have demonstrated little efficacy for relief of neuropathic pain. ${ }^{13}$ In fact, many SSRIs are less effective than TCAs in the treatment of diabetic peripheral neuropathic pain., ${ }^{5,14}$ The side effects of SSRIs include nausea, diarrhea, constipation, sleep disturbance, and sexual dysfunction. ${ }^{13}$ There is evidence that suggests that dual serotonin and NE reuptake inhibitors 
are more effective for the relief of neuropathic pain than the SSRIs. ${ }^{15}$ One of the first selective serotonin and NE reuptake inhibitors (SSNRIs), venlafaxine, has been shown to lower neuropathic pain intensity. However, the difficulty with using venlafaxine in clinical practice is that it is predominantly a SSRI at low doses and only has dual SNRI properties at higher doses, which are often required for pain relief but are accompanied by side effects such as insomnia, irritability, dizziness, and cardiac palpitations. 5,15,16

Duloxetine hydrochloride (duloxetine) and pregabalin are currently approved by the Food and Drug Administration (FDA) for the management of diabetic peripheral neuropathic pain. In addition, duloxetine is also used for the treatment of major depressive disorder (MDD), generalized anxiety disorder, and fibromyalgia. ${ }^{17-20}$ In addition, it is approved in Europe for the treatment of stress urinary incontinence. ${ }^{21}$ Duloxetine was also found in clinical trials to significantly reduce the severity of painful physical symptoms that are associated with depression. ${ }^{19,20}$ Analysis showed that over half of the reduction in painful physical symptoms was due to true pain reduction as a direct effect from duloxetine and not a secondary effect due to improvement in mood..$^{20,22}$ In light of the finding that duloxetine effectively reduced painful physical symptoms in MDD and evidence that serotonin and NE are important modulators of descending inhibitory pain pathways in the central nervous system, duloxetine was examined for pain relief in patients with neuropathic pain due to diabetic peripheral neuropathy without coexisting depression. ${ }^{20,22}$

Duloxetine is a potent SSNRI that has little affinity for other receptors or ion channels in the nervous system. ${ }^{5}$ Animal studies have shown that duloxetine is a more potent inhibitor of serotonin and NE reuptake than other SNRIs, which include venlafaxine and milnacipran. ${ }^{5}$ It is also a relatively balanced dual reuptake inhibitor of both serotonin and NE. Animal studies in which rats were depleted of serotonin demonstrated that duloxetine was as efficacious as paroxetine, a SSRI, in blocking depletion of serotonin content. In addition, in rats depleted of NE, duloxetine was as efficacious as thionisoxetine and desipramine, which are selective norepinephrine reuptake inhibitors (NRIs), in blocking the depletion of NE concentrations. ${ }^{5}$

Animal models of acute and persistent pain demonstrated that duloxetine is efficacious in relieving persistent pain without resulting in significant neurological deficits. ${ }^{23}$ However, it was found to be less efficacious in relieving acute pain. ${ }^{5}$ This finding was explained based on the different underlying pathologic mechanisms behind acute and persistent pain. In the animal model used for acute pain, the tail-flick test, there is no tissue or nerve damage and the pain response mainly involves a spinally mediated reflex. In contrast, the animal models of persistent pain, the formalin model and L5/L6 nerve ligation, involve tissue and nerve injury. These injuries lead to hyperexcitabiltiy of neurons in the spinal cord and supraspinal pain pathways, which is referred to as central sensitization. ${ }^{5}$ Central sensitization is due to an imbalance between the endogenous inhibitory and excitatory pain pathways. Therefore, a medication such as duloxetine that blocks the reuptake of serotonin and NE, which are both key mediators of the descending pain pathways, should be effective in relieving persistent neuropathic pain. ${ }^{5}$

Duloxetine is absorbed beginning 2 hours after oral administration and the maximum plasma concentration is achieved in approximately 6 hours. Taking duloxetine with food increases the time to peak absorption by 6 to 10 hours, but it does not effect its maximum plasma concentration. In addition, administration in the evening compared to the morning results in a 3-hour delay in the absorption and increases clearance by approximately $33 \%{ }^{13}$

Duloxetine is metabolized rapidly by cytochrome P-450 enzymes and has a half-life of approximately 12 hours. Over $70 \%$ of the metabolites are excreted in the urine and the remaining $20 \%$ are excreted in the feces. None of duloxetine's metabolites have been found to contribute significantly to its pharmacologic activity. ${ }^{13}$ There are no recommendations to adjust the dose of duloxetine when it is given with other medications that are metabolized by the cytochrome P-450 enzymes. However, use of duloxetine in combination with a monoamine oxidase inhibitor (MAOI) medication is contraindicated and duloxetine should not be started until 14 days after discontinuing therapy with an MAOI. This recommendation is due to the risk of serious or fatal reactions reported in patients taking serotonergic medications in combination with a MAOI. These reactions include the serotonin syndrome, which includes the following signs: hyperthermia, rigidity, myoclonus, autonomic instability, and mental status changes that may progress to delirium or coma. ${ }^{13}$ The use of duloxetine is also contraindicated in patients with uncontrolled narrow-angle glaucoma.

Precaution should be used if duloxetine is used in patients with hepatic insufficiency and it should not be used in patients with a substantial history of alcohol use or evidence of chronic liver disease. In patients with hepatic dysfunction the mean plasma clearance is $15 \%$ of that observed in patients with normal liver function and the half-life increases 3-fold. In addition, duloxetine is not recommended in patients with a creatinine clearance of less than $30 \mathrm{~mL} / \mathrm{min} .{ }^{13}$ 
Duloxetine is a highly protein-bound medication ( $>90 \%)$. To date, the interactions between duloxetine and other highly protein-bound medications have not been evaluated. Therefore it is recommended that either adding or removing duloxetine to an existing medication regimen be done with caution if there is a concern for an effect on protein binding. In addition, duloxetine is a pregnancy category $\mathrm{C}$ medication and it is recommended that the dose may need to be tapered and the drug discontinued in pregnant women. ${ }^{13}$

\section{Efficacy studies with duloxetine in neuropathic pain}

Clinical studies have shown that duloxetine is effective and safe in the management of diabetic peripheral neuropathic pain. ${ }^{6,15,20}$ To date, there have been three randomized, doubleblind, multicenter, placebo-controlled trials to assess the efficacy and safety of duloxetine (Table 1). All three trials were 12-week acute therapy trials. Enrollment criteria included the presence of a bilateral, symmetric neuropathy associated with either type 1 or type 2 diabetes mellitus that caused daily pain for at least 6 months. The mean average daily pain severity had to be at least 4 on an 11-point Likert scale $(0=$ no pain, $10=$ worst pain possible). The primary efficacy endpoint in these studies was either a $30 \%$ or $50 \%$ reduction in a 24-hour average pain score on the Likert scale. This endpoint was chosen because previous studies involving patients with chronic pain syndromes have shown that an average reduction of 2 or more points, or approximately $30 \%$ from baseline on an 11-point pain rating scale correlates with a clinically meaningful improvement as determined by the 7-point patient global impression of change. In fact, a $30 \%$ change in the pain intensity numerical rating scale correlated with a patient global impression category of "much improved" and a 50\% change corresponded to "very much improved". ${ }^{24}$

All three trials demonstrated a statistically significant reduction in pain severity for duloxetine $60 \mathrm{mg}$ once a day (60 mg/day) and $60 \mathrm{mg}$ twice a day (120 mg/day) compared to placebo. There was no significant difference in efficacy seen between these two treatment groups. ${ }^{6,15,20}$ The study by Goldstein et al included a duloxetine $20 \mathrm{mg}$ once-a-day treatment group, and found that this group had a nonsignificant decrease in pain severity compared to placebo. ${ }^{20}$

The significant reduction in pain for the duloxetine $60 \mathrm{mg} /$ day and $120 \mathrm{mg} /$ day treatment groups was seen beginning in the first week of therapy and it persisted throughout the length of the study. ${ }^{20}$ Even though most of the responses to duloxetine occurred in the first 1 to 2 weeks of treatment, a potential benefit to continuing therapy with duloxetine was observed. Specifically, of those patients that did not have a significant response to duloxetine after 6 weeks of therapy, approximately one-third achieved a significant response after the remaining 12 weeks of treatment. ${ }^{25}$

A pooled analysis of these three studies found that approximately two-thirds of patients who were treated with duloxetine achieved a $30 \%$ or greater reduction in 24-hour average pain severity. Using the alternative criteria of a 2-point reduction on the 11-point pain rating scale, approximately $60 \%$ of the patients treated with duloxetine achieved this clinically meaningful improvement in pain severity. Applying the more stringent criteria of a $50 \%$ or greater reduction in 24-hour average pain severity, approximately half of all duloxetine-treated patients achieved a response compared to $28 \%$ of those receiving placebo. ${ }^{25}$ The response rates, defined as $\geq 50 \%$ pain reduction, were $48.2 \%$ (120 mg/day), 47.2\% (60 mg/day), and 27.9\% (placebo). ${ }^{3}$

Secondary measures of pain were also significantly improved in both the duloxetine $60 \mathrm{mg} /$ day and $120 \mathrm{mg} /$ day treatment groups compared to placebo. ${ }^{15,20}$ These secondary measures included worst pain, night pain, the Brief Pain Inventory (BPI) average pain severity, BPI worst pain severity, BPI least pain severity, BPI pain severity right now, and the Short-Form McGill Pain Questionnaire (SF-MPQ) total score. ${ }^{20}$ The patients in the duloxetine $20 \mathrm{mg}$ once-a-day treatment group had significant improvements compared to placebo on measures of worst pain score and the SF-MPQ total score. ${ }^{20}$ In addition, further analysis revealed that there was a significantly higher percentage of patients taking duloxetine $120 \mathrm{mg} /$ day who reported improvement in shooting, stabbing, sharp, hot-burning, and splitting pain sensations compared to patients in the placebo group. ${ }^{20}$

The pooled data from the three placebo-controlled studies of duloxetine was also examined to determine the impact of baseline disease variables related to diabetes and diabetic neuropathy severity on the efficacy of duloxetine. There were no significant effects of age, type of diabetes, duration of diabetes, duration of diabetic neuropathy, severity of diabetic neuropathy as measured by the Michigan Neuropathy Screening Instrument, or baseline hemoglobin $\mathrm{A}_{1 \mathrm{C}}$ on the efficacy of duloxetine. However, it was found that duloxetine was more effective in patients with higher initial pain scores (BPI average pain of 6 or greater). ${ }^{26}$

Another measure of efficacy for pain relief that was examined in the clinical trials was the average daily dose of supplemental analgesic medication used. Goldstein et al performed a comparison that demonstrated that patients 


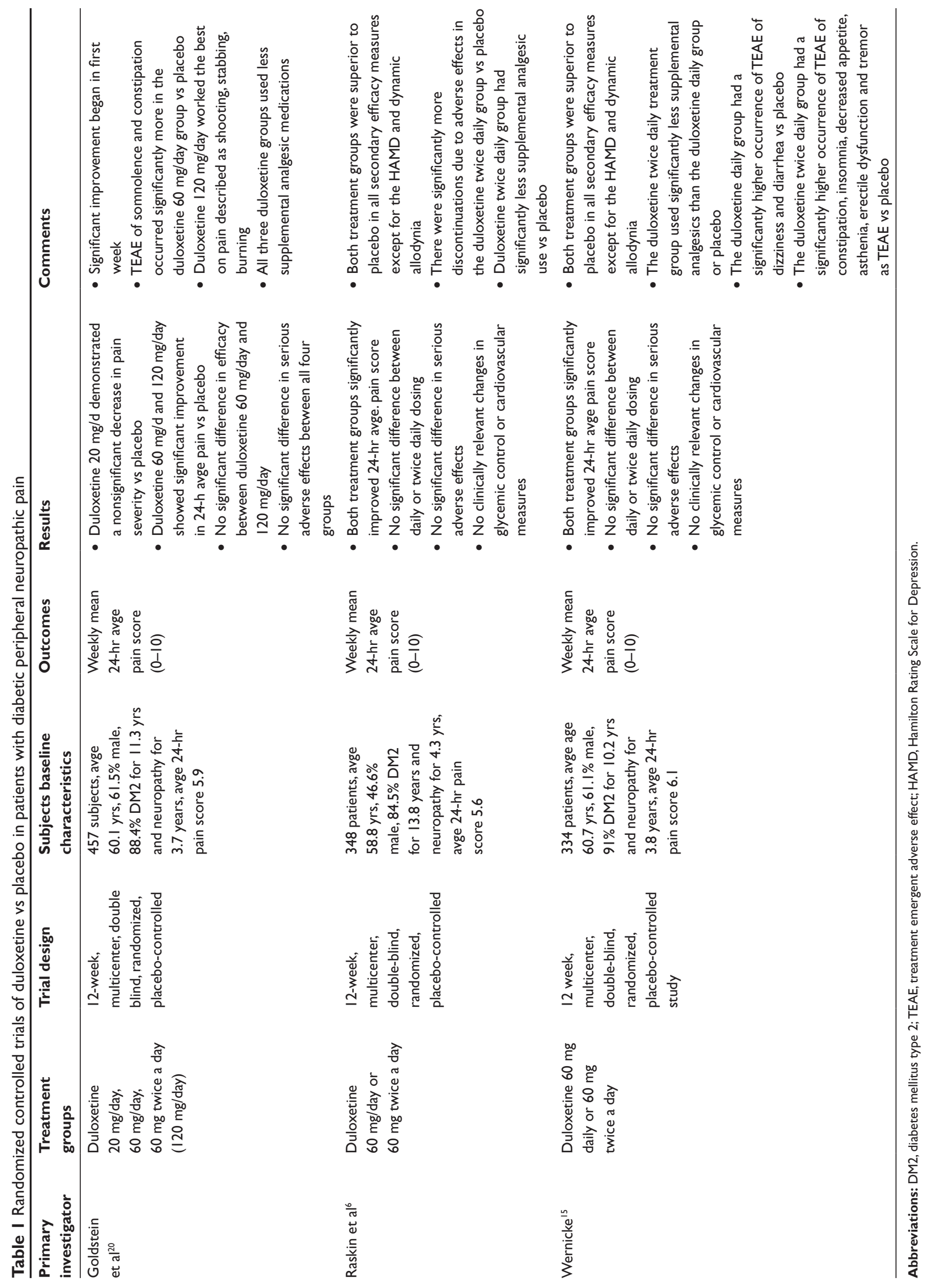


who were treated with duloxetine at any dose $(20 \mathrm{mg} /$ day, $60 \mathrm{mg} /$ day, or $120 \mathrm{mg} /$ day) required a lower average daily dose of supplemental analgesic medication compared to patients who received placebo. ${ }^{20}$ The duloxetine $60 \mathrm{mg} /$ day and $120 \mathrm{mg} /$ day treatment groups used significantly less analgesic medication than the placebo group and the duloxetine $20 \mathrm{mg} /$ day group used numerically less than the placebo group. ${ }^{20}$ In 2 other studies it was found that only patients treated with duloxetine $120 \mathrm{mg} /$ day took significantly less supplemental analgesic medication than patients treated with either duloxetine $60 \mathrm{mg} /$ day or placebo. ${ }^{6,15}$ This result provides evidence of additional efficacy of duloxetine $120 \mathrm{mg} /$ day compared to $60 \mathrm{mg} /$ day, however there is also an increase in adverse effects. ${ }^{22}$

An open-label study by Skljarevski et al that enrolled 216 patients concluded that the effect of duloxetine $60 \mathrm{mg}$ /day in patients with diabetic peripheral neuropathic pain was maintained over a longer, 6-month treatment period. ${ }^{23}$ In addition, there was an arm in the study for patients who were nonresponders to therapy with duloxetine $60 \mathrm{mg} /$ day. After 8 weeks of therapy with duloxetine $60 \mathrm{mg}$ /day, patients who did not report at least a $30 \%$ decrease in 24-hour average pain were then treated with duloxetine $120 \mathrm{mg} /$ day for the remainder of the study, which was 26 weeks. This study found that 115 patients $(53 \%)$ responded to duloxetine $60 \mathrm{mg} /$ day and were put into the maintenance arm. The remaining participants, the nonresponders, who received an increased dose of duloxetine $120 \mathrm{mg}$ /day, reported a statistically significant reduction in their 24-hour average pain. Findings from this study suggest that treatment with duloxetine $120 \mathrm{mg}$ /day provides an additional $10 \%$ of patients with a clinically significant pain reduction of $50 \% .^{11}$

Data derived from a meta-analysis and original publications of duloxetine vs pregablin and gabapentin in the treatment of diabetic peripheral neuropathic pain is shown in Table $2 .{ }^{11}$ The primary endpoints for efficacy in pain control were reduction in 24-hour pain severity, response rate of $50 \%$ or greater pain reduction, and Patient Global Impression of Improvement/Change (PCI-I/C). This meta-analysis concluded that duloxetine had comparable efficacy and tolerability to gabapentin and pregabalin in the treatment of diabetic peripheral neuropathic pain. It was determined that all three drugs were superior to placebo for all three efficacy measures. ${ }^{11}$

In the study by Quilici et al pooled analysis estimated that the number needed to treat for improvement in pain was 5 (95\% confidence interval [CI] 3 to 7 ) for duloxetine and 5 (95\% CI 4 to 8 ) for pregabalin. ${ }^{11} \mathrm{~A}$ different pooled analysis of 6 placebo-controlled trials of duloxetine estimated the number needed to treat with duloxetine as 5.9 (95\% CI 4.8 to 7.7$).{ }^{17}$ Other reviews have reported the number needed to treat for gabapentin as 3.4 or 3.8, but this does not represent a direct comparison. Furthermore, the number needed to treat for the TCAs has previously been estimated at approximately 3 , but these trials were small crossover trials that may overestimate the efficacy of TCAs. ${ }^{11}$

A recent systematic review of treatments for diabetic peripheral neuropathic pain found that oral tricyclic antidepressants and traditional anticonvulsants were better for short-term pain relief than newer-generation anticonvulsants. ${ }^{8}$ The classes of drugs included in this study were paracetamol (acetaminophen), antidepressants, opioids, nonsteroidal anti-inflammatory drugs, N-methyl-D-aspartate antagonists, tramadol, capsaicin, and anticonvulsants. All drugs were compared to placebo and efficacy was defined as a $50 \%$ or greater reduction in pain. This study found that oral tricyclic antidepressants, anticonvulsants (sodium valproate, lamotrigine, carbamazepine), and opioids had better efficacy than newer-generation anticonvulsants (oxcarbazepine, pregabalin, gabapentin), a selective serotonin reuptake inhibitor (citalopram), and duloxetine. ${ }^{8}$ Shortcomings of this review include the fact that it is difficult to compare outcomes, especially when related to pain, if different primary endpoints are used. In addition, sample sizes of the included trials were small, the treatment period was less then 6 months in all the studies, and some of the trials used a crossover design without a washout period. In the studies with a crossover design there may have been selection bias because only data from the first period was used to calculate efficacy. Furthermore, some medications had multiple studies included in the analysis, some of which had contradictory results, and other medications may have only had 1 study included. It is clear that further, long-term and head-to-head studies are needed to examine the efficacy of commonly used pharmacological treatments for diabetic peripheral neuropathic pain.

Although treatment with duloxetine has a significant effect on diabetic peripheral neuropathic pain, it has not been found to alter peripheral nervous system pathology or result in regeneration of peripheral nerve axons. In studies of duloxetine there were no treatment group differences in any electrophysiologic measures of nerve function, including nerve conduction studies of the ulnar and peroneal nerves. ${ }^{6,15}$

\section{Safety and tolerability of duloxetine}

In all of the clinical studies, the incidence of serious adverse events associated with treatment with duloxetine was low and 
Table 2 Indirect comparison of neuropathic pain results: duloxetine vs pregabalin

\begin{tabular}{|c|c|c|c|}
\hline Outcome & $\begin{array}{l}\text { Mean difference } \\
\text { in treatment effects }\end{array}$ & $\begin{array}{l}95 \% \text { confidence } \\
\text { interval }\end{array}$ & Comments \\
\hline $\begin{array}{l}\text { Mean reduction in } \\
\text { 24-hour pain score }\end{array}$ & -0.248 & $0.667 ; 0.162$ & $\begin{array}{l}\text { Duloxetine was not inferior to } \\
\text { pregabalin }\end{array}$ \\
\hline $\begin{array}{l}\text { Patient global impression } \\
\text { of change }\end{array}$ & 0.542 & $0.016 ; 1.060$ & $\begin{array}{l}\text { Pregabalin was slightly more } \\
\text { effective than duloxetine }\end{array}$ \\
\hline $\begin{array}{l}\text { Premature discontinuation } \\
\text { due to lack of efficacy }\end{array}$ & -0.251 & $-1.288 ; 0.717$ & No statistical difference found \\
\hline $\begin{array}{l}\text { Premature discontinuation } \\
\text { due to adverse events }\end{array}$ & 0.152 & $-0.505 ; 0.790$ & No statistical difference found \\
\hline Diarrhea & 0.886 & $-0.4 \mid 4 ; 2.183$ & No statistical difference found \\
\hline Dizziness & -1.084 & $-1.903 ;-0.317$ & $\begin{array}{l}\text { Duloxetine produced a significantly } \\
\text { lower incidence of dizziness }\end{array}$ \\
\hline Headache & 0.700 & $-0.078 ; 1.458$ & No statistical difference found \\
\hline Somnolence & -0.554 & $-1.458 ; 0.328$ & No statistical difference found \\
\hline
\end{tabular}

Note: Derived from data of. ${ }^{6,11,15,20,33-37}$

the medication was generally well tolerated. ${ }^{6,15,20}$ Reported side effects, which were typically mild to moderate in severity, included nausea, fatigue, somnolence, increased sweating, dry mouth, dizziness, diarrhea, constipation, insomnia, decreased appetite, asthenia, erectile dysfunction, weakness, and tremor. ${ }^{6,11,15,17}$

In the study by Goldstein et al less than $20 \%$ of subjects discontinued participation in the trial because of adverse events. There was not a significant difference in reported treatment-emergent adverse events (TEAE) in the duloxetine $20 \mathrm{mg} /$ day treatment group compared to placebo. There were more discontinuations due to adverse events in the duloxetine $60 \mathrm{mg} /$ day and $120 \mathrm{mg} /$ day treatment groups compared to the placebo group. However, the only TEAEs that occurred significantly more frequently in the duloxetine $60 \mathrm{mg}$ /day treatment group compared to placebo were somnolence and constipation. Except for somnolence, which was reported as severe in the duloxetine $120 \mathrm{mg}$ /day treatment group, most of the reported adverse effects were mild to moderate in severity. ${ }^{20}$

Other trials by Raskin et al and Wernicke et al also found that there was a significant increase in reported TEAEs in the duloxetine $60 \mathrm{mg}$ /day and $120 \mathrm{mg} /$ day treatment groups. ${ }^{6,15}$ These events were also generally mild to moderate in severity. Patients in both duloxetine treatment groups reported a significant increase in treatment-emergent nausea, somnolence, hyperhydrosis and anorexia compared to placebo. Furthermore, patients in the duloxetine $120 \mathrm{mg} /$ day treatment group had significantly more frequent vomiting and constipation than patients in the placebo group. Compared to placebo, there were significantly more patients in the duloxetine $120 \mathrm{mg} /$ day treatment group who discontinued therapy due to TEAEs. Adverse events that lead to discontinuation of the medication in at least $1 \%$ of duloxetine treated patients were nausea, vomiting, dizziness, somnolence, and fatigue. ${ }^{6,15}$

A longer-term study of patients with diabetic peripheral neuropathic pain examined the safety of duloxetine $120 \mathrm{mg}$ /day compared to routine care for up to 52 weeks. ${ }^{27}$ It was found that there were no significant differences in the occurrence of serious adverse events. The one TEAE with a significant group difference was asthenia, which occurred at a higher frequency in patients treated with duloxetine. ${ }^{27}$

Pooled analysis of trials of duloxetine in diabetic peripheral neuropathic pain have found that treatment with duloxetine leads to a significantly higher incidence of dizziness, nausea, headache, and somnolence. ${ }^{11}$ These TEAEs typically occur at the beginning of drug therapy and resolved over time. ${ }^{20,28}$ Furthermore, most patients who discontinued duloxetine due to adverse events did so within the first 4 weeks of treatment. ${ }^{6}$ An explanation for this may be that these trials typically start patients on $60 \mathrm{mg}$ of duloxetine once a day and after 3 days increased the dose to $60 \mathrm{mg}$ twice a day. A slower titration schedule may likely decrease the incidence of TEAEs. In fact, studies in patients with major depressive disorder suggested that either starting patients on a dose of duloxetine $30 \mathrm{mg}$ once a day for a week or starting $60 \mathrm{mg}$ in the morning with food may reduce the incidence of nausea compared to a starting dose of $60 \mathrm{mg}$ once a day. ${ }^{18}$

Another question on the administration of duloxetine in clinical practice is once daily vs twice daily dosing. 
A 28-week open label safety study of duloxetine $60 \mathrm{mg}$ twice daily or $120 \mathrm{mg}$ once daily found that both doses were safe and well tolerated for the duration of the trial. ${ }^{28}$ There were few differences in safety or tolerability between the two doses and both doses provided clinically significant reduction of pain. However, there were more discontinuations due to adverse effects in the duloxetine $120 \mathrm{mg}$ once daily arm, which suggests that a dosing regimen of duloxetine $60 \mathrm{mg}$ twice daily may be better tolerated.

One study found that some TEAEs, such as back pain, arthralgias, and pruritis, were reported significantly less frequently by the duloxetine $60 \mathrm{mg} /$ day and $120 \mathrm{mg} /$ day treatment groups compared to placebo. In addition, the patients in the placebo group experienced a significantly greater incidence of lower limb edema and peripheral swelling than the duloxetine treated patients. ${ }^{20}$

A meta-analysis of duloxetine vs pregablin and gabapentin in the treatment of diabetic peripheral neuropathic pain found that treatment with duloxetine, pregabalin, or gabapentin resulted in a significantly higher rate of discontinuation due to adverse events compared to placebo. The most commonly reported TEAEs for both pregabalin and gabapentin were dizziness and somnolence and duloxetine treatment resulted in significantly higher reports of dizziness, headache, nausea, and somnolence than placebo. However, duloxetine produced a significantly lower incidence of dizziness compared to pregabalin. ${ }^{11}$

In all of the studies of duloxetine for the treatment of diabetic peripheral neuropathic pain there were several serious adverse events reported. Serious adverse events were defined as any event resulting in or prolonging hospitalization or death, a life threatening experience, or severe or permanent disability. There were no significant group differences in the occurrence of serious adverse events between the duloxetine treatment groups and placebo and none of the serious adverse events were determined to be due to treatment with duloxetine. . $^{6,15,20}$

There have been case reports of patients developing hepatic failure while taking duloxetine. The incidence of this occurrence is approximately 1 to 2 per 100,000 exposures and it is more common in patients with a history of significant alcohol use and/or chronic liver disease. ${ }^{29}$ In addition, a small fraction of patients treated with duloxetine have been found to have elevations in their liver enzymes that resolve spontaneously. Overall the hepatic risk of duloxetine has been determined to be within the range for other medications and does not require monitoring of hepatic enzymes. ${ }^{29}$ However, it is recommended that duloxetine should not be prescribed to patients with substantial alcohol use or evidence of chronic liver disease and it should be discontinued in patients who develop jaundice or other evidence of clinically significant liver dysfunction.

In addition to adverse events, there were no clinically meaningful changes in laboratory data including blood chemistry, hematology, glycemic control, or lipid levels with duloxetine treatment. ${ }^{6,15,20}$ Due to duloxetine's noradrenergic potentiation, careful measurements of blood pressure, heart rate, and the QTc interval on electrocardiogram were monitored. Studies of duloxetine have found that there is an average increase of $2 \mathrm{mmHg}$ in diastolic blood pressure and an elevation in baseline heart rate of approximately 2 to 3 beats $/ \mathrm{min}$. ${ }^{30}$ These findings were significant when compared to placebo but were not determined to be clinically significant. In addition, duloxetine was not found to adversely affect the QTc interval, which can be prolonged by medications such as TCAs, ${ }^{6,15,20}$ or systolic blood pressure even during a year-long safety study. ${ }^{27}$ These findings led to the conclusion that patients with diabetes who are treated with duloxetine do not require additional cardiovascular monitoring other than what is indicated for treatment of their underlying diabetes. ${ }^{6,15,20,28}$

\section{Duloxetine and quality of life measures in neuropathy}

The impact that treatment with duloxetine had on quality of life measures in patients with diabetic peripheral neuropathic pain were included in the clinical trials with measures of general illness severity and improvement as well as health outcome scores, which were examined as secondary measures. Overall there were significant improvements in most measures of quality of life in addition to pain reduction due to treatment with duloxetine. ${ }^{15,20}$ The duloxetine $60 \mathrm{mg} /$ day and $120 \mathrm{mg} /$ day groups had significant improvements in measures of general illness severity and improvement, as measured by the Clinical Global Impression of Severity and Patients Global Impression of Improvement, and the duloxetine $20 \mathrm{mg}$ /day group had significant improvements in the Clinical Global Impressions of Severity. ${ }^{20}$

Health outcome measures were evaluated by use of patient completed questionnaires. These included the BPI-Interference, Euro Quality of Life instrument (EQ-5D), and the Short Form-36 (SF-36) Health Status Survey. The BPI-Interference measures how much pain interferes with several patient outcomes including general activity, mood, walking, ability, sleep, and interpersonal relationships. The EQ-5D measures how severe the patient perceived general health. The SF-36 measures how a patient perceives their general status and 
consists of 36 items that calculate 8 health domains: bodily pain, general health, mental health, physical functioning, physical role, emotional role, social function, and vitality.

Pooled analysis from the three randomized, double-blind, multicenter, placebo-controlled trials of duloxetine in diabetic peripheral neuropathic pain demonstrated that patients treated with duloxetine improved significantly on all health outcome measures compared to placebo. The measures of functional outcomes had robust improvements that were unaffected by either the time or incidence of patient withdrawal. ${ }^{4}$ A study comparing duloxetine with routine care in patients with diabetic peripheral neuropathic pain found a significant group difference in the SF-36 physical component summary score and subscale scores of physical functioning, bodily pain, mental health, and vitality in patients who were treated with duloxetine $60 \mathrm{mg}$ twice a day compared to those who received routine care. ${ }^{27}$

Previous studies have shown that patients with diabetes consistently score lower than age matched individuals without the disease on patient-rated measures of quality of life and that diabetes is associated with deficits in health-related quality of life. ${ }^{4}$ The result that treatment with duloxetine results in improvement in health outcome measures suggests that treatment with duloxetine was associated with a significant improvement in daily functioning. This emphasizes the clinical role of duloxetine in management of patients with diabetic peripheral neuropathic pain and its potential to significantly improve quality of life in these patients.

The question arises whether the improvement in measures of quality of life seen with duloxetine therapy is due to its effect as an antidepressant or due to a reduction in neuropathic pain. In addition, it is unclear how much pain reduction can be attributed to improvements in mood and what effect on mood duloxetine may have on patients who do not have MDD. For this reason, patients who met criteria within the past year for the Diagnostic and Statistical Manual of Mental Disorders, Fourth Edition (DSM-IV) Axis I diagnosis of MDD, dysthymic disorder, generalized anxiety disorder, alcohol or eating disorders were excluded from enrollment in the duloxetine studies of efficacy in diabetic peripheral neuropathic pain. To screen for subclinical depression, changes in mood and anxiety were measured at enrollment and at the end of the trials with either the Hamilton Rating Scale for Depression (HAMD) or the Beck Depression Inventory-II and the Beck Anxiety Inventory.

It was found that there were no significant changes in mood or anxiety between groups treated with duloxetine and placebo. ${ }^{6,15,20}$ In addition, the mean change in mood from baseline to endpoint as measured with the HAMD was very similar in the placebo and treatment groups. ${ }^{6}$ This finding was explained based on the exclusion criteria and the fact that baseline measures of depression and anxiety were low in these subjects, with little measurable room for improvement and it suggests that duloxetine has a neutral effect on mood for patients who do not have a clinical diagnosis of depression. ${ }^{6,15,20}$ Further analysis showed that more than $90 \%$ of the reduction in pain was due to a direct effect on pain modulation and not due to an antidepressant effect of duloxetine. .,30 $^{6}$

\section{Conclusion: the place of duloxetine in the treatment of diabetic neuropathic pain}

Duloxetine is approved by the FDA specifically for the treatment of diabetic peripheral neuropathic pain. It has been proven to be superior to placebo at doses of $60 \mathrm{mg} /$ day and $120 \mathrm{mg} /$ day in treating diabetic peripheral neuropathic pain and its effect of reducing 24-hour average pain is seen early, typically within the first week. Comparison studies have shown that its efficacy is comparable to that of other medications commonly used for the treatment of diabetic peripheral neuropathic pain. Duloxetine is relatively well tolerated. However, lower titration schedules may improve tolerability. Greater pain efficacy may be attained with doses higher than $60 \mathrm{mg} /$ day in patients who do not respond to the recommended maintenance dose. Clinically relevant changes in health outcome measures that affect quality of life have also been found to improve with therapy.

In addition to safety and efficacy, the cost of medications is a major concern for patients and clinicians. A recent study by Beard et al examined the cost-effectiveness of duloxetine in the treatment of diabetic peripheral neuropathic pain in the UK. The standard approach to treatment of diabetic peripheral neuropathy in the UK is to use a TCA as a first-line trial of treatment. Other medications such as anticonvulsants are then tried before using narcotics for pain control. Given this paradigm, they found that the second line use of duloxetine is a beneficial and cost-effective treatment strategy for diabetic peripheral neuropathic pain. ${ }^{12}$ A similar study in the US examined the relative efficacy, costs, and cost effectiveness of first-line treatment options for painful diabetic neuropathy. This study found that desipramine and duloxetine were both more effective and less expensive than gabapentin and pregabalin. ${ }^{31}$ In comparison with pregabalin, it was found that even though duloxetine and pregabalin had comparable levels of a full response (at least a $50 \%$ reduction in pain), treatment with pregabalin resulted in a greater proportion of patients 
with a partial response (30\% to $49 \%$ reduction in pain). Prior studies have demonstrated clear differences in health utility between full and partial pain response that impacts the cost-effectiveness of alternative treatments. Patients taking duloxetine would be less likely to have a partial pain response and require additional therapy with other costly medications. ${ }^{12}$ Therefore, duloxetine is an acceptable initial or alternative treatment for patients with diabetic neuropathic pain.

Unfortunately, treatment algorithms for painful diabetic neuropathy are based upon clinical experience and pooled data from trials with relatively small sample sizes, nonstandardized markers of efficacy, and short follow-up studies. Furthermore, there is a lack of data from head-to-head comparison trials, which prohibits true evaluation of the safety and efficacy of widely used neuropathic pain agents. Trials are needed that use 1) head-to-head comparisons or individual neuropathic pain medication or combinations of pain medications, 2) longer durations of follow-up, and 3) use of standardized and validated pain assessment scales to allow accurate comparison of data across trials. ${ }^{32}$

\section{Acknowledgments}

This work was supported in part by The American Diabetes Association (ADA), the Juvenile Diabetes Research Foundation (JDRF), NIH RR024888, Office of Research Development (Medical Research Service), Department of Veterans Affairs (JWR).

\section{Disclosures}

The authors declare no conflicts of interest.

\section{References}

1. Cowie CC, Rust KF, Ford ES, Eberhardt MS, Byrd-Holt DD, Li C, et al. Full accounting of diabetes and pre-diabetes in the US population in 1988-1994 and 2005-2006. Diabetes Care. 2009;32:287-294.

2. Gaster B, Hirsch IB. The effects of improved glycemic control on complications in type 2 diabetes. Arch Int Med. 1998;158:134-140.

3. Ziegler D. Treatment of diabetic neuropathy and neuropathic pain: how far have we come? Diabetes Care. 2008;31 Suppl 2:S255-S261.

4. Armstrong DG, Chappell AS, Le TK, Kajdasz DK, Backonja M, D'Souza DN, et al. Duloxetine for the management of diabetic peripheral neuropathic pain: evaluation of functional outcomes. Pain Med. 2007;8:410-418.

5. Iyengar S, Webster AA, Hemrick-Luecke SK, Xu JY, Simmons RM. Efficacy of duloxetine, a potent and balanced serotonin-norepinephrine reuptake inhibitor in persistent pain models in rats. $J$ Pharmacol Exp Ther. 2004;311:576-584.

6. Raskin J, Pritchett YL, Wang F, D’Souza DN, Waninger AL, Iyengar S, et al. A double-blind, randomized multicenter trial comparing duloxetine with placebo in the management of diabetic peripheral neuropathic pain. Pain Med. 2005;6:346-356.

7. Writing Team for the Diabetes Control and Complications Trial/ Epidemiology of Diabetes Interventions and Complications Research Group. Effect of intensive therapy on the microvascular complications of type 1 diabetes mellitus. JAMA. 2002;287:2563-2569.
8. Wong MC, Chung JW, Wong TK. Effects of treatments for symptoms of painful diabetic neuropathy: systematic review. BMJ. 2007;335:87.

9. Smith AG, Russell JW, Feldman EL, Goldstein J, Peltier A, Smith S, et al. Lifestyle intervention for prediabetic neuropathy. Diabetes Care. 2006; 29:1294-1299.

10. Gaede P, Vedel P, Parving HH, Pedersen O. Intensified multifactorial intervention in patients with type 2 diabetes mellitus and microalbuminuria: the Steno type 2 randomised study. Lancet. 1999;353:617-622.

11. Quilici S, Chancellor J, Lothgren M, Simon D, Said G, Le TK, et al. Meta-analysis of duloxetine vs pregabalin and gabapentin in the treatment of diabetic peripheral neuropathic pain. BMC Neurol. 2009;9:6.

12. Beard SM, McCrink L, Le TK, Garcia-Cebrian A, Monz B, Malik RA. Cost effectiveness of duloxetine in the treatment of diabetic peripheral neuropathic pain in the UK. Curr Med Res Opin. 2008;24:385-399.

13. Westanmo AD, Gayken J, Haight R. Duloxetine: a balanced and selective norepinephrine- and serotonin-reuptake inhibitor. Am J Health Syst Pharm. 2005;62:2481-2490.

14. Max MB, Lynch SA, Muir J, Shoaf SE, Smoller B, Dubner R. Effects of desipramine, amitriptyline, and fluoxetine on pain in diabetic neuropathy. $N$ Engl J Med. 1992;326:1250-1256.

15. Wernicke JF, Pritchett YL, D'Souza DN, Waninger A, Tran P, Iyengar S, et al. A randomized controlled trial of duloxetine in diabetic peripheral neuropathic pain. Neurology. 2006;67:1411-1420.

16. Ginsberg DL. Venlafaxine Effective for Painful Polyneuropathy. Prim Psychiatry. 2003;10:41.

17. Sultan A, Gaskell H, Derry S, Moore RA. Duloxetine for painful diabetic neuropathy and fibromyalgia pain: systematic review of randomised trials. BMC Neurol. 2008;8:29.

18. Whitmyer VG, Dunner DL, Kornstein SG, Meyers AL, Mallinckrodt CH, Wohlreich MM, et al. A comparison of initial duloxetine dosing strategies in patients with major depressive disorder. J Clin Psychiatry. 2007;68:1921-1930.

19. Goldstein DJ, Lu Y, Detke MJ, Hudson J, Iyengar S, Demitrack MA. Effects of duloxetine on painful physical symptoms associated with depression. Psychosomatics. 2004;45:17-28.

20. Goldstein DJ, LuY, Detke MJ, Lee TC, Iyengar S. Duloxetine vs. placebo in patients with painful diabetic neuropathy. Pain. 2005;116:109-118.

21. Ghoniem GM, Van Leeuwen JS, Elser DM, Freeman RM, Zhao YD, Yalcin I, et al. A randomized controlled trial of duloxetine alone, pelvic floor muscle training alone, combined treatment and no active treatment in women with stress urinary incontinence. J Urol. 2005;173:1647-1653.

22. Russell JW. Duloxetine in painful diabetic neuropathy. Nat Clin Pract Neurol. 2006;2:18-19.

23. Skljarevski V, Desaiah D, Zhang Q, Chappell AS, Detke MJ, Gross JL, et al. Evaluating the maintenance of effect of duloxetine in patients with diabetic peripheral neuropathic pain. Diabetes Metab Res Rev. 2009. [Epub ahead of print].

24. Farrar JT, Young JP Jr, LaMoreaux L, Werth JL, Poole RM. Clinical importance of changes in chronic pain intensity measured on an 11-point numerical pain rating scale. Pain. 2001;94:149-158.

25. Pritchett YL, McCarberg BH, Watkin JG, Robinson MJ. Duloxetine for the management of diabetic peripheral neuropathic pain: response profile. Pain Med. 2007;8:397-409.

26. Ziegler D, Pritchett YL, Wang F, Desaiah D, Robinson MJ, Hall JA, et al. Impact of disease characteristics on the efficacy of duloxetine in diabetic peripheral neuropathic pain. Diabetes Care. 2007;30: 664-669.

27. Wernicke JF, Wang F, Pritchett YL, Smith TR, Raskin J, D’Souza DN, et al. An open-label 52-week clinical extension comparing duloxetine with routine care in patients with diabetic peripheral neuropathic pain. Pain Med. 2007;8:503-513.

28. Raskin J, Wang F, Pritchett YL, Goldstein DJ. Duloxetine for patients with diabetic peripheral neuropathic pain: a 6-month open-label safety study. Pain Med. 2006;7:373-385.

29. Wohlreich MM, Acharya N, Strombom I, Kuritzky L, Robinson M, Heinloth AN, et al. Answers to the most common questions about the hepatic safety profile of duloxetine. Postgrad Med. 2008;120:111-118. 
30. Kroenke K, Krebs EE, Bair MJ. Pharmacotherapy of chronic pain: a synthesis of recommendations from systematic reviews. Gen Hosp Psychiatry. 2009;31:206-219.

31. O'Connor AB, Noyes K, Holloway RG. A cost-utility comparison of four first-line medications in painful diabetic neuropathy. Pharmacoeconomics. 2008;26:1045-1064.

32. Murad MH, Smith SA. Review: tricyclic antidepressants, anticonvulsants, opioids, and capsaicin cream are effective treatments for diabetic neuropathy. ACP J Club. 2008;148:2.

33. Rosenstock J, Tuchman M, LaMoreaux L, Sharma U. Pregabalin for the treatment of painful diabetic peripheral neuropathy: a double-blind, placebo-controlled trial. Pain. 2004;110:628-638.

34. Lesser H, Sharma U, LaMoreaux L, Poole RM. Pregabalin relieves symptoms of painful diabetic neuropathy: a randomized controlled trial. Neurology. 2004;63:2104-2110.
35. Freynhagen R, Strojek K, Griesing T, Whalen E, Balkenohl M. Efficacy of pregabalin in neuropathic pain evaluated in a 12-week, randomised, double-blind, multicentre, placebo-controlled trial of flexible- and fixed-dose regimens. Pain. 2005;115:254-263.

36. Richter RW, Portenoy R, Sharma U, LaMoreaux L, Bockbrader H, Knapp LE. Relief of painful diabetic peripheral neuropathy with pregabalin: a randomized, placebo-controlled trial. J Pain. 2005;6:253-260.

37. Tolle T, Freynhagen R, Versavel M, Trostmann U, Young JP Jr. Pregabalin for relief of neuropathic pain associated with diabetic neuropathy: a randomized, double-blind study. Eur J Pain. 2008;12:203-213.

\section{Publish your work in this journal}

Diabetes, Metabolic Syndrome and Obesity: Targets and Therapy is an international, peer-reviewed open-access journal committed to the rapid publication of the latest laboratory and clinical findings in the fields of diabetes, metabolic syndrome and obesity research. Original research, review, case reports, hypothesis formation, expert opinion and commentaries are all considered for publication. The manuscript management system is completely online and includes a very quick and fair peer-review system, which is all easy to use. Visit http://www.dovepress.com/testimonials.php to read real quotes from published authors.

Submit your manuscript here: http://www.dovepress.com/diabetes-metabolic-syndrome-and-obesity-targets-and-therapy-journal 